\title{
Understanding the gut microbiome of dairy calves: Opportunities to improve early-life gut health ${ }^{1}$
}

\author{
Nilusha Malmuthuge ${ }^{2}$ and Le Luo Guan ${ }^{3}$ \\ Department of Agricultural, Food and Nutritional Science, University of Alberta, Edmonton, AB, T6G 2P5 Canada
}

\begin{abstract}
Early gut microbiota plays a vital role in the longterm health of the host. However, understanding of these microbiota is very limited in livestock species, especially in dairy calves. Neonatal calves are highly susceptible to enteric infections, one of the major causes of calf death, so approaches to improving gut health and overall calf health are needed. An increasing number of studies are exploring the microbial composition of the gut, the mucosal immune system, and early dietary interventions to improve the health of dairy calves, revealing possibilities for effectively reducing the susceptibility of calves to enteric infections while promoting growth. Still, comprehensive understanding of the effect of dietary interventions on gut microbiotaone of the key aspects of gut health - is lacking. Such knowledge may provide in-depth understanding of the mechanisms behind functional changes in response to dietary interventions. Understanding of host-microbial interactions with dietary interventions and the role of the gut microbiota during pathogenesis at the site of infection in early life is vital for designing effective tools and techniques to improve calf gut health.
\end{abstract}

Key words: microbiome, microbiota, early-life gut health, calves

\section{INTRODUCTION}

The role of the gut microbiome (the microbial taxonomic composition and their collective genomes) in the development and function of the gastrointestinal tract and in gut health across animal species has been widely recognized. The presence of gut microbiota is necessary for the development of the intestinal epithelium,

\footnotetext{
Received November 4, 2016.

Accepted April 4, 2017.

${ }^{1}$ Presented as part of the Non-Nutrition: The Future of Nutrition? Symposium at the ADSA-ASAS Joint Annual Meeting, Salt Lake City, Utah, July 2016.

${ }^{2}$ Current address: Vaccine and Infectious Disease Organization, University of Saskatchewan, Saskatoon, S7N 5E3 Canada.

${ }^{3}$ Corresponding author: lguan@ualberta.ca
}

the mucosal layer (Sharma et al., 1995), and lymphoid structures (Mebius, 2003), as well as for the differentiation of immune cell repertoire (Smith and Garrett, 2011). The use of germ-free rodent models has successfully explained the role of gut microbiota in modulating the anatomy and physiology of the mammalian gastrointestinal tract and intestinal-mucosa-associated immune system (Sommer and Bäckhed, 2013). A recent attempt to cross-colonize human gut microbiota in mice suggested that only host-specific microbiota could trigger the maturation of the mucosal immune system (Chung et al., 2012); it is therefore important to study host-microbial interactions that affect host health within a species.

Although the human gut microbiome has received the most attention due to its immense importance for human health, more and more studies are investigating the importance of gut microbiota in the health and production of livestock (Kogut and Arsenault, 2016). Cattle provide milk and meat to meet growing demands for animal proteins as the human population increases. They can convert low-quality dietary substrates that are unsuitable for human consumption into high-quality animal protein (meat and milk) through sustainable farming (Eisler et al., 2014). However, the cattle industry is facing many challenges - one of them being the high rates of preweaned calf mortality (USDA, 2010) - that affect replacement herds. Enteric infections in neonatal calves are one of the major causes of calf death (Uetake, 2013), despite preventive measures. Moreover, with the new regulations limiting the prophylactic use of antimicrobials (Smith, 2015), the need for alternative approaches to minimize diarrhea incidence in neonatal calves is urgent.

Methods to improve calf gut health during the preweaning period are necessary to minimize calves' susceptibility to enteric infections. Gut health is an ambiguous term used to describe multiple factors that contribute to maintaining disease-free status in the gastrointestinal tract (Bischoff, 2011). Manipulation of the gut microbiome, a key factor that influences gut health (Bischoff, 2011), is one option for improving calf gut health. However, a better understanding of 
host-microbial interactions in the preweaned calf gut is needed to design effective microbial manipulation tools and techniques. The aim of this review was to summarize available information on the gut microbiota and mucosal immune system of preweaned dairy calves and critically evaluate the microbial manipulation methods currently in use to improve calf gut health to identify knowledge gaps that need to be addressed to improve dairy calf health.

\section{ASSESSMENT OF GUT MICROBIOTA: WHERE ARE WE?}

More research into the gut microbiota of dairy calves has become available with the development of cultureindependent molecular-based approaches. Among them, next-generation sequencing (NGS) has become affordable for many researchers as a way of including microbial analysis in their studies. However, not all studies provide clear definitions of terms, especially in animal nutrition research. For example, microbiota and microbiome are often used interchangeably, but microbiota is the composition of a microbial community, usually identified by targeted sequencing of $16 \mathrm{~S}$ rRNA gene using amplicon sequencing, and microbiome is the total genetic information of a microbial community (microbiota), which can be identified using whole-genome sequencing of the metagenome.

In the past, a targeted or desired region of the $16 \mathrm{~S}$ rRNA gene was sequenced by using Sanger sequencing, after cloning of PCR products (Chakravorty et al., 2007; Tringe and Hugenholtz, 2008). With the development of NGS approaches that use bridge amplification (Illumina sequencing) or emulsion amplification (454 sequencing, SOLiD) of a single DNA strand, cloning of PCR products is no longer needed (Shendure and Ji, 2008). As well, NGS approaches generate highthroughput data by sequencing a large number of pooled samples after adding identifiers or barcodes, and they drastically decrease the cost of sequencing (Tringe and Hugenholtz, 2008). Of the NGS approaches, gut microbiologists prefer Roche 454 pyrosequencing and Illumina sequencing (Arrieta et al., 2014), which are associated with well-developed bioinformatics pipelines for community composition analysis such as quantitative insight into microbial ecology (QIIME) and mothur (Plummer et al., 2015).

In addition to targeted 16SrRNA amplicon-based sequencing, NGS can be used to sequence the genomic DNA and messenger RNA of a microbial community to study the metagenome and the metatranscriptome of a gut microbiome, respectively. They allow study of the functions of microbial communities while generat- ing taxonomic composition data. Metagenomics-based approaches can be used to explore microbial gene composition and functional abundance; metatranscriptomics-based approaches can be used to investigate the active microbiome by measuring levels of gene expression (Hugenholtz and Tyson, 2008). The advantage of studying the metatranscriptome over the metagenome is that it allows for exploration of the active microbial community. Amplicon sequencing has been used widely to study the gut microbiota of preweaned calves as a way of identifying their composition (presence or absence of particular taxa) and how they can be affected by age, diet, or growth (Oikonomou et al., 2013; KleinJöbstl et al., 2014; Malmuthuge et al., 2014; Foditsch et al., 2015). To date, metagenome sequencing to explore the function of gut microbiota in preweaned calves is rare (Malmuthuge, 2016), and no attempts have been made to study the active microbial community of dairy calves using a metatranscriptome-based approach.

\section{PREWEANED CALF GUT MICROBIOME: WHAT DO WE KNOW?}

The calf gut microbiota has been studied mainly using fecal samples (Uyeno et al., 2010; Mayer et al., 2012; Oikonomou et al., 2013; Klein-Jöbstl et al., 2014; Tomassini, 2015), but a few studies have used local intestinal samples (Malmuthuge et al., 2012, 2014). These studies have reported the presence of a simple, less diverse bacterial community at birth that increases in complexity and diversity with growth, as a result of age and dietary changes (Uyeno et al., 2010; Mayer et al., 2012; Oikonomou et al., 2013; Klein-Jöbstl et al., 2014; Tomassini, 2015; Dill-McFarland et al., 2017). The first colonizers identified from calf meconium samples are Citrobacter, Lactococcus, Leuconostoc, and Lactobacillus, and meconium microbial composition is very similar to the fecal microbiota at 6 and $12 \mathrm{~h}$ after birth (Mayer et al., 2012). However, the similarity between fecal microbial composition and meconium samples decreases drastically after $24 \mathrm{~h}$ of life because of increased diversity (Mayer et al., 2012), indicating the establishment of a complex microbiome very early in life. Individual variations observed among calves decrease with calf age (Klein-Jöbstl et al., 2014), suggesting the establishment of a more similar microbial community in older calves. Bacterial groups detected in the fecal samples of preweaned calves revealed increasing or decreasing patterns in their relative abundance with calf growth. For example, the abundance of bacterial genera Bifidobacterium, Lactobacillus, Fecalibacterium, and Enterococcus decreases with growth (Uyeno et al., 2010; Oikonomou et al., 2013; Klein-Jöbstl et 
al., 2014; Tomassini, 2015). Although these studies report similar patterns of gut microbial colonization using fecal samples, variations among studies in gut microbial composition (the relative abundance of detected bacterial groups) are evident. The majority of studies reported a higher abundance of genus Bacteroides (phylum Bacteroidetes) in the fecal samples of preweaned calves (Uyeno et al., 2010; Klein-Jöbstl et al., 2014; Tomassini, 2015). Oikonomou et al. (2013) reported a higher abundance of Firmicutes in the samples collected through rectal swabs within the first $6 \mathrm{wk}$ of life; however, the authors did not discuss bacterial composition at the genus level in detail. Foditsch et al. (2015) also reported a higher abundance of Firmicutes in rectal swab samples, and genus-level information revealed that Bacteroides was one of the predominant bacterial groups. The contrasting results observed among studies may have resulted from differences in microbial profiling methods, breeds used, and feeding methods or diet. Mayer et al. (2012) showed that the fecal microbial composition was more similar between twin calves than between siblings, implying that host genetics partly define individual gut microbial composition. Thus, the use of different calves raised under varying management practices may have also contributed to the observed differences among studies.

Gut microbial colonization during the preweaning period has primarily been studied using fecal samples, because it is a noninvasive way to collect samples from the same individual over time. However, Malmuthuge et al. (2014) reported that bacterial composition could vary depending on the gastrointestinal tract region and sample type used (content versus mucosal tissue). Firmicutes and Bacteroidetes dominated the bacterial communities along the gastrointestinal tract of preweaned calves at $3 \mathrm{wk}$ of life, but the relative abundance of these 2 phyla varied greatly among local gut regions and between the tissue and content within each region.

Most studies focused on the composition of the gut bacteria, but a few reported the composition of archaea in the gut of preweaned calves (Zhou et al., 2014; DillMcFarland et al., 2017). Although gut bacteria have been studied mainly to understand their modulatory roles in the mucosal immune system and in calf health (Malmuthuge et al., 2012; Oikonomou et al., 2013), studies on the intestinal archaeal community emphasize that in-depth understanding of this microbial group is useful in controlling enteric methane emissions (Zhou et al., 2014; Guzman et al., 2015; Dill-McFarland et al., 2017). Similar to gut bacteria, the archaea in the calf gut vary along the gastrointestinal tract (Zhou et al., 2014) and display high individual variation in their composition (Zhou et al., 2014; Dill-McFarland et al., 2017).

\section{EFFECT OF PREWEANED CALF DIET ON GUT MICROBIAL COMPOSITION}

In addition to studies describing the gut microbial composition of preweaned dairy calves, a few studies describe the effects of diet on this composition. The preweaned calf diet is changed rapidly from milk or milk replacer to a solid diet within a few weeks of birth, and the industry uses various management strategies to optimize the transition and weaning process (Khan et al., 2011). Existing studies have mainly explored the effect of calf starter (Malmuthuge et al., 2013), transition or weaning methods (Meale et al., 2016), and feeding methods (Maynou et al., 2016) on gut microbial composition in preweaned calves. Supplementation of a milk replacer diet with calf starter during weaning tended to increase the number of bacterial phylotypes (a group of $16 \mathrm{~S}$ gene sequences sharing more similarities to each other) in the calf gut, although it did not affect bacterial density or the proportion of lactic acid bacteria in calf small intestine (Malmuthuge et al., 2013). Those authors noted that milk replacer accounted for the majority of the diet during weaning, and this might have masked the effect of starter on gut microbiota. Another attempt to study the effect of transition or weaning method on the fecal microbiota of dairy calves revealed no differences in bacterial composition between abrupt and gradual weaning (Meale et al., 2016). Once again, calves were on a diet that consisted mainly of milk replacer, regardless of their weaning method, implying that dietary differences were not significant enough to influence gut microbial composition. A comparison of the fecal microbiota of calves fed pasteurized waste milk or milk replacer revealed a lower prevalence of bacterial orders Clostridales and Bacteroidales in calves fed pasteurized waste milk than in calves fed milk replacer (Maynou et al., 2016). Although the effect of the preweaned calf diet has been well studied in terms of calf rumen development and the weaning process, we know less about how it influences gastrointestinal tract microbiota and microbial interactions with host functions. The early introduction of calf starter promotes rumen development and facilitate early weaning (Khan et al., 2011), but it may also influence the intestinal gut barrier and immune responses by altering the expression of genes associated with microbial recognition and barrier functions (Malmuthuge et al., 2013).

\section{ANTIMICROBIAL USE DURING EARLY LIFE AND GUT MICROBIAL COMPOSITION}

Antimicrobials are used heavily in the dairy industry to promote gut health and lower the risk of enteric infections (Smith, 2015). However, their use in food 
animal production is a controversial issue, with studies reporting low effectiveness in preventing enteric infections (Smith, 2015). As well, increasing public awareness of antimicrobial resistance has led to new regulations limiting the prophylactic use of antimicrobials in dairy calves (Thames et al., 2012). A few attempts have been made to understand the effect of antimicrobials on gut microbial composition (Xie et al., 2013; Oultram et al., 2015; Pereira et al., 2016) and the occurrence of genes for antimicrobial resistance in calf gut microbiota (Khachatyran et al., 2004; Thames et al., 2012; Maynou et al., 2016, 2017).

Treating calves with the antimicrobial bacitracin methylene disalicylate (BMD) altered the fecal microbial composition of calves by increasing the number of opportunistic pathogens such as Escherichia, Enterococcus, and Shigella, and decreasing beneficial bacteria such as Roseburia, Faecalibacterium, and Eubacterium (Xie et al., 2013). This increase in potentially pathogenic bacterial groups was observed in all calves treated with BMD regardless of their health status, but in calves that did not receive antibiotic treatment, the increase was observed only when they had diarrhea (Xie et al., 2013). Use of antibiotics to treat calf diarrhea and respiratory diseases has also led to alterations in the fecal microbial composition of preweaned calves (Oultram et al., 2015). The abundance of Lactobacillus decreased with all antibiotic treatments, but the greatest reduction in Lactobacillus abundance was observed with oxytetracycline, a broad-spectrum antibiotic (Oultram et al., 2015). In a recent study, feeding milk that contained residual antimicrobials influenced the fecal microbiota of preweaned calves (Pereira et al., 2016). The relative abundance of Clostridium and Streptococcus decreased when calves were fed milk that contained antimicrobials. These observed changes in gut bacterial composition following antimicrobial exposure indicates that antimicrobials cause dysbiosis (microbial imbalance) in the gut microbiota of preweaned calves. Maynou et al. (2016) reported that feeding waste milk containing $\beta$-lactam antimicrobial residues increased the presence of $\beta$-lactamase resistance genes in the Escherichia coli population of preweaned calves compared to calves fed milk replacer. In another study, Maynou et al. (2017) reported a higher number of antimicrobial-resistant (enrofloxacin, florfenicol, streptomycin) E. coli phenotypes in calves fed waste milk than in calves fed milk replacer. The prevalence of antimicrobial-resistant fecal $E$. coli phenotypes was also influenced by calf age, regardless of feeding program (Maynou et al., 2017). For example, enrofloxacin- and doxycycline-resistant genes increased in abundance during the first 6 wk of life, but doxycycline-resistant genes decreased from 6 wk to 1 yr of age (Maynou et al., 2017). These studies indicate that early antimicrobial treatments influence the microbial composition and the prevalence of antimicrobial genes in the gut microbiota of preweaned calves, and the occurrence of antimicrobial-resistant genes in the gut microbiota can change with calf age. We may see further decreases in the effectiveness of the antimicrobials used to treat preweaned calves with infectious diseases because of increases in the abundance of antimicrobial resistance genes in the early gut microbiome.

\section{MUCOSAL IMMUNE SYSTEM IN PREWEANED CALVES}

Besides preserving a healthy gut microbiome, maintaining effective immune responses that can detect, prevent, and eliminate invading pathogens while tolerating commensal microbiota is a key aspect of gut health (Bischoff, 2011). The mucosal immune system comprises physical barriers (mucosal layer, epithelium), chemical barriers (antimicrobial peptides, secretory IgA) and pattern-recognition receptors [toll-like receptors (TLR), NOD-like receptors], as well as a repertoire of immune cells involved in performing the above tasks (Hooper et al., 2012). Physical barriers play a crucial role by preventing the invasion of microbiota present in the intestinal lumen into intestinal tissue enriched with immune cells. The mucosal layer, the first physical barrier, contains secreted net-like glycosylated mucins that trap microbiota (Johansson et al., 2011). The secretory compounds (IgA, antimicrobial peptides) also limit the growth of microbiota on the mucosal layer (Johansson et al., 2011). For example, secreted IgA in response to commensal microbiota is non-inflammatory and provides protection by binding to microbiota entering the mucosal layer (Gutzeit et al., 2014). Beneath the mucosal layer, a single layer of epithelial cells, connected by an intracellular junctional complex known as tight junctions (Ulluwishewa et al., 2011), regulates the movement of macromolecules through the layer. Our recent studies on the whole transcriptome (Liang et al., 2016) and microRNAome of the small intestine (Liang et al., 2014) of preweaned dairy calves have provided clues about the molecular mechanisms that regulate intestine development in early life. Profiling of the microRNAome, the regulatory molecules of gene expression, revealed regional and temporal variations in the expression of detected microRNA in the jejunum and ileum of preweaned calves (Liang et al., 2014). The main difference in the expression of microRNA was observed during the first week of life, and the functions of these differentially expressed microRNA were related to the development of intestinal epithelial cells (Liang et al., 2014). When those authors studied the transcriptome of the same calves, they found that expression of 
tight-junction protein coding genes (claudin 1, claudin 4 , and occludin) upregulated during the first week of life (Liang et al., 2016). These findings suggest that that the first week of life is crucial for the development of intestinal epithelium or the physical barrier of the mucosal immune system. The expression of tightjunction protein coding genes was also affected by the addition of calf starter to a diet of milk replacer during weaning, indicating increased gut permeability with starter feeding (Malmuthuge et al., 2013). Future studies are needed to determine how different nutritional strategies affect barrier function, which is so important for protecting the host from microbial invasions and absorbing nutrients.

However, when we compared 2 regions of the small intestine in preweaned calves, the jejunum had more tight-junction protein coding genes than the ileum ( $\mathrm{Li}-$ ang et al., 2016), indicating poor barrier function at the ileum. We also observed differences between the jejunum and the ileum in the expression of genes related to B cell lineage (Liang et al., 2016). Differences in the lymphoid structures and their functions have also been reported between the jejunum and the ileum of fetal and postnatal calves (Yasuda et al., 2006). The development of follicles and the proliferation of $\mathrm{B}$ cells in the ileal Peyer's patches were different than in the jejunum, and these differences implied that the jejunum acts as an immune induction site, but the ileum does not (Yasuda et al., 2006). The use of an RNAseq approach (transcriptome profiling) by Liang et al. (2016) further confirmed that the jejunum has greater immune induction capacity than the ileum.

Moreover, exploring the mucosal leukocyte populations of the small intestines of preweaned calves ( 3 to 5 wk of age) revealed significant differences in the distribution and abundance of leukocyte populations between the jejunum and the ileum (Fries et al., 2011a). $\mathrm{A}$ dendritic cell subpopulation $\left(\mathrm{CD} 11 \mathrm{c}^{\mathrm{Hi}} \mathrm{CD} 14^{+}\right)$and natural killer cells $\left(\mathrm{CD} 335^{+}\right)$were highly abundant in the ileum but not in the jejunum, and macrophages were observed only in the ileal intraepithelial compartment (Fries et al., 2011a). Studying the myeloid and lymphoid cell populations in the small intestine revealed a higher abundance of some myeloid cell populations (CD4, CD8, and $\gamma \sigma \mathrm{TcR} \mathrm{T}$ cells, and CD11cHiCD14+ macrophages) in the ileum of weaned calves than in the jejunum, but these differences were not observed in preweaned calves (Fries et al., 2011b). Apart from the observed regionality, Fries et al. (2011b) also reported significant differences in mucosal leukocyte populations between preweaned and weaned calves. Total mucosal leukocytes $\left(\mathrm{CD} 45^{+}\right)$increased in both gut regions as calves got older, but CD4 cells and natural killer cells were significantly higher in the jejunum in weaned calves than in preweaned calves (Fries et al., 2011b). Age-dependent changes in mucosal immune cell populations can also be different between the 2 small intestinal regions. These findings suggest regional differences between the jejunum and ileum exist at various levels of system biology, such as immune cell populations (Fries et al., 2011a) in the anatomy of the lymphoid structures (Yasuda et al., 2006) and at the molecular level (Liang et al., 2014, 2016).

A comparison of the expression of TLR and antimicrobial peptides ( $\beta$-defensin, peptidoglycan recognition protein 1) between preweaned and weaned calves mainly revealed an age-dependent expression pattern (Malmuthuge et al., 2012). No differences were observed in the expression of genes between the jejunum and the ileum, except for TLR1, TLR9, and TLR10 in preweaned calves and TLR10 in weaned calves, which showed higher expression in the ileum than in the jejunum (Malmuthuge et al., 2012). Interestingly, Fries et al. (2011b) and Malmuthuge et al. (2012) used the same animals to study immune cell populations and the expression of immune-related genes. These studies together suggested a decrease in the innate immune-related response (decrease in TLR expression in Malmuthuge et al., 2012) and an increase in adaptive immune response (increase in mucosal $\mathrm{T}$ cells in Fries et al., 2011b) with increasing calf age. A switch from innate immunity to adaptive immunity with age has also been shown in humans, and has been suggested as a sign of a developed mucosal immune system (Teran et al., 2011).

\section{OPPORTUNITIES TO IMPROVE CALF GUT HEALTH VIA THE GUT MICROBIOME}

The gut microbiome plays a crucial role in the development, maturation, and homeostasis of the mucosal immune system (Hooper et al., 2012), and dysbiosis in the gut microbiome has been linked to various enteric disorders that cause intestinal tissue inflammation in humans and mice (Sartor and Mazmanian, 2012). Maintaining normal bacterial densities and composition is necessary for a healthy gut (Bischoff, 2011); thus, nurturing a healthy gut microbiome to improve gut health and overall host health is a topic of interest for humans and livestock.

An overgrowth in E. coli has been observed in the small intestines of calves with neonatal calf diarrhea (Constable, 2004), suggesting dysbiosis in gut microbiota. However, it is not clear whether this dysbiosis is the cause or the result of enteric infections. Oikonomou et al. (2013) have suggested that the early gut microbial composition of calves might be linked to susceptibility to enteric infections, indicating that individual varia- 
tions in early gut microbiota may play a role in the pathogenesis of neonatal calf diarrhea. A recent study attempted to improve calf health by manipulating early gut microbial composition with oral supplementation of Faecalibacterium prausnitzii (Foditsch et al., 2015), a bacterium reported to be negatively associated with calf diarrhea (Oikonomou et al., 2013). Oral administration of $F$. prausnitzii during the first week of life effectively reduced the incidence of diarrhea and calf death related to diarrhea in preweaned calves during the first 7 wk of life (Foditsch et al., 2015). As well, F. prausnitzii was more abundant in treated calves during the first 5 wk of life than in control calves (Foditsch et al., 2015), indicating that early microbial interventions persist within the gut microbiome, and that this persistence may play a role in influencing host health. Thus, this study showed promising results for early microbial intervention to improve calf gut health and overall health. Besides understanding the effects of microbial manipulations on host health, it is important to understand their effect on the gut microbiome, the potential modulators of gut health and host health. However, the authors did not observe statistical differences in the relative abundance of the detected bacterial groups, except for Faecalibacterium, which was higher in the treated group than in the controls (Foditsch et al., 2015). A lack of information about variations in the relative abundance of different bacterial groups prevents us from understanding the contribution of individual animal differences to these observations. Studies on human gut (Yatsunenko et al., 2012) and rumen (Jami et al., 2013) microbiomes have revealed that individual animal variation is higher in early life than in adulthood, and this might have contributed to the lack of statistical differences between bacterial groups. Moreover, information is lacking on the gut microbiome of calves with diarrhea to understand what makes them susceptible to enteric infections even if they are treated. In-depth understanding of the gut microbiome using metagenomics and metatranscriptomics is needed to help us clarify microbial-microbial interactions that lead to dysbiosis during enteric infections in preweaned calves.

Failure of passive transfer of immunity due to poor colostrum management leads to high incidence of diarrhea (Lorenz et al., 2011). Therefore, timed feeding of high-quality colostrum (IgG $>50 \mathrm{mg} / \mathrm{mL}$ of colostrum) is an important good calf management practice for preventing neonatal calf diarrhea (Godden, 2008). Feeding heat-treated colostrum has been reported to decrease diarrhea incidence in calves compared with those fed fresh colostrum (Godden et al., 2012). One of our recent studies revealed that feeding colostrum soon after birth facilitated bacterial colonization in the small intestine in dairy calves within $12 \mathrm{~h}$ of life, compared with calves that did not receive colostrum within 6 or $12 \mathrm{~h}$ after birth (Malmuthuge et al., 2015). This study did not investigate the long-term effects of different colostrumfeeding methods on gut microbiota. However, a single feeding of heat-treated colostrum soon after birth promoted colonization with Bifidobacterium and reduced colonization with E. coli, suggesting that colostrum acts as a natural prebiotic that promotes the establishment of beneficial bacteria in the preweaned calf gut. Such an increase in Bifidobacterium and a decrease in E. coli may have been one reason behind the decreased incidence of diarrhea in calves fed heat-treated colostrum in the Godden et al. (2012) study. Hence, we need to understand the role of colostrum, besides being immunogenic, in establishing a gut microbiome that interacts with the developing mucosal immune system. Such knowledge will provide opportunities to improve gut health through early microbial manipulation.

Although their effect on gut microbiota have not been evaluated, feeding yeast (Saccharomyces cerevisiae) cultures or yeast products to improve health has been tested in preweaned calves. Feeding yeast products decreases the number of days with diarrhea in calves that have failure of passive transfer of immunity (Galvão et al., 2005). Moreover, feeding yeast cultures with grain decreases the incidence of diarrhea and the mortality rate in calves within the first $70 \mathrm{~d}$ of life (Magalhães et al., 2008). A recent study evaluated the effect of yeast products on immune response in pathogen infections (Citrobactor freundii, Salmonella enterica) and revealed that calves supplemented with yeast products had a lower neutrophil-to-lymphocyte ratio and lower fecal scores (Harris, 2016). The authors suggested that this finding might have been due to a decrease in inflammatory response, equivalent to fewer signs of infection and better gut health. Together, these studies suggest that yeast cultures and yeast products can be used to decrease the susceptibility of calves to enteric infections. However, they overlooked the effect of feeding microbiota or microbial products on the gut microbiome. Feeding the fermentation products of yeast to dairy calves altered rumen microbial composition during the experimental period, but not at the end of the feeding trial (Xiao et al., 2016). When the authors profiled the microbiota of the duodenum at the end of the trial, they found no changes in microbial composition between control calves and calves supplemented with the fermentation products of yeast (Xiao et al., 2016). This study further reported that small intestinal morphology changed at the end of the trial with supplementation of the fermentation products of yeast, but did not provide details on the small intestinal gut microbiome. Thus, a few questions need to be answered to understand the 
effect of yeast or yeast products on calf gut health: (1) What are the factors that drive morphological changes in the small intestine of calves supplemented with yeast or yeast products? (2) Do the changes observed in the duodenum represent what is happening in the jejunum and ileum? and (3) Is starting treatment 1 month after birth too late to influence the gut microbiome that has already been established?

Quercetin, a flavonoid, has been tested on calves to evaluate if it can affect the plasma immunoglobulin level of colostrum-deprived calves that are highly susceptible to enteric infections (Gruse et al., 2016). Flavonoids are bioactive compounds that are beneficial for human health because of their antioxidant, anti-inflammatory, and antimicrobial activities (Bell, 2016). However, quercetin could not improve plasma immunoglobulin levels to the level observed in calves fed colostrum (Gruse et al., 2016). In addition, lower levels of plasma glucose and total protein, and higher levels of C-reactive proteins and serum amyloid (indicators of inflammation), were observed in the colostrum-deprived calves (Gruse et al., 2016), and these blood parameters remained the same when the colostrum-deprived calves were supplemented with quercetin (Gruse et al., 2016). However, quercetin has been shown to promote balanced gut microbial composition in rats fed a diet high in fat and sucrose that altered microbial composition by increasing the ratio of Firmicutes to Bacteroidetes (Etxeberria et al., 2015). Supplementing the rats with quercetin for 6 wk decreased the abundance of phylum Firmicutes and class Erysipelotrichi, but did not lead to differences in the blood parameters tested (insulin, glucose, $\mathrm{TNF} \alpha$, lipopolysaccharides; Etxeberria et al., 2015). In contrast, Gruse et al. (2016) looked only at the shortterm effects $(9 \mathrm{~d})$ of quercetin on blood parameters and did not evaluate its effect on the gut microbiome. Thus, we cannot yet conclude whether feeding quercetin is an effective dietary manipulation method for calves.

\section{THE FUTURE}

Based on mouse models and in vitro studies, we know that the gut microbiota modulates the expression of TLR that recognize microbial patterns and prime the immune system (Rakoff-Nahoum et al., 2004), and that play a vital role in maintaining the integrity of the intestinal barrier by interacting with tight-junction protein coding genes (Ulluwishewa et al., 2011). In our previous studies (Malmuthuge et al., 2012; Liang et al., 2014, 2016), we reported associations between bacterial densities and expression of immune-related genes that imply interactions among early gut microbiota and host mucosal immune system development. Enteric pathogens in neonatal calves mainly target the small intestine as the major site of infection (Cho and Yoon, 2014). Because it has a lower barrier function (Liang et al., 2016) and low immune induction capacity (Yasuda et al., 2006), and because it is frequently targeted by enteric pathogens (Cho and Yoon, 2014), the ileum is an ideal gut region in which to study host-microbial interactions and explore opportunities for improving calf gut health.

Based on our previous study (Malmuthuge et al., 2015), it is clear that the ileum is colonized with a dense bacterial population at birth, and that feeding heat-treated colostrum soon after birth alters bacterial composition by promoting colonization with Bifidobacterium and decreasing colonization with E. coli (Malmuthuge et al., 2015). Therefore, we need to study the interactions between ileal microbiota and ileal mucosal immune components when using microbial manipulation methods, to generate an in-depth understanding of the mechanisms that promote calf gut health. This will be fundamental to designing effective manipulation tools and techniques.

Studies on maternal effects on the establishment of the early gut microbiome, such as maternal microbiota (Dominguez-Bello et al., 2010) and maternal stress during pregnancy (Jasarevic et al., 2015; Zijlmans et al., 2015), are being conducted in humans and mice. These factors may cause epigenetic changes (chemical changes that occur in DNA without modifying the DNA sequence of a gene) and are known as epigenetic stimuli that influence the behavior, health, and metabolism of the offspring (Pacchierotti and Spanò, 2015). Maternal antibiotic treatment has been shown to influence the early gut microbiome of offspring by altering the microbiome of the birth canal (Stokholm et al., 2014), one of the early exposures for newborns. Antimicrobials are often used in the dairy industry to treat cows (Léger et al., 2017); therefore, understanding maternal effects on the early gut microbiome and gut health of preweaned calves will also be important.

\section{CONCLUSIONS}

High calf mortality caused by enteric infections, as well as increasing pressure to decrease the use of prophylactic antimicrobials, encourage multidisciplinary approaches to improve gut health in neonatal calves by manipulating the gut microbiome. However, our understanding of the microbiome in the development of mucosal immune system and maintenance of immune homeostasis is very limited. Although the number of studies is increasing, only a few studies have tried to integrate understanding of gut microbiota and host health; most studies focus on these factors separately. The majority of studies used fecal samples to sequence 
16S rRNA genes and explore gut microbial composition, but this approach does not allow us to understand the functionality of the gut microbiome. Studies comparing the bacterial composition of the gastrointestinal tract in neonatal calves have reported regional differences in the gut, as well as differences between tissue-attached and content-associated bacteria. Therefore, we need to focus on host-microbial interactions at the site of enteric infection (ileum) in the future. Huge individual variations in calf gut microbial composition have been reported, but attempts to understand the effects of host genetics or epigenetics are lacking. Moreover, dietary manipulations have been tested to decrease diarrhea incidence and calf mortality, but these studies have not explored modulatory mechanisms or effects on the gut microbiome. We need to use integrated approaches to understand host-microbial interactions in the pathogenesis of enteric infections and during dietary interventions to improve calf health by manipulating the gut microbiome.

\section{ACKNOWLEDGMENTS}

The authors acknowledge financial support from a Natural Science Engineering Research Council (NSERC) discovery grant and Alberta Livestock Meat Association (ALMA) to L. L. Guan, and from an Alberta Innovates Doctoral Graduate Student Scholarship and Banting Postdoctoral Fellowship to N. Malmuthuge.

\section{REFERENCES}

Arrieta, M. C., L. T. Stiemsma, N. Amenyogbe, E. M. Brown, and B. Finlay. 2014. The intestinal microbiome in early life: Health and disease. Front. Immunol. 5:427 https://doi.org/10.3389/ fimmu.2014.00427.

Bell, J. S. 2016. Effects of a flavonoid-rich diet on gut microbiota composition and production of trimethylamine in human subjects. MS Thesis. Utah State University, Logan.

Bischoff, S. C. 2011. 'Gut health': A new objective in medicine? BMC Med. 9:24. https://doi.org/10.1186/1741-7015-9-24.

Chakravorty, S., D. Helb, M. Burday, N. Connell, and D. Alland. 2007. A detailed analysis of $16 \mathrm{~S}$ ribosomal RNA gene segments for the diagnosis of pathogenic bacteria. J. Microbiol. Methods 69:330-339.

Cho, Y. I., and K.-J. Yoon. 2014. An overview of calf diarrhea-infectious etiology, diagnosis, and intervention. J. Vet. Sci. 15:1-17. https://doi.org/10.4142/jvs.2014.15.1.1.

Chung, H., S. J. Pamp, J. A. Hill, N. K. Surana, S. M. Edelman, E. B. Troy, N. C. Reading, E. J. Villablanca, S. Wang, J. R. Mora, Y. Umesaki, D. Mathis, C. Benoist, D. A. Relman, and D. L. Kasper. 2012. Gut immune maturation depends on colonization with a host-specific microbiota. Cell 149:1578-1593. https://doi. org/10.1016/j.ce11.2012.04.037.

Constable, P. D. 2004. Antimicrobial use in the treatment of calf diarrhea. J. Vet. Intern. Med. 18:8-17.

Dill-McFarland, K. A., J. D. Breaker, and G. Suen. 2017. Microbial succession in the gastrointestinal tract of dairy cows from 2 weeks to first lactation. Sci. Rep. 7:40864 https://doi.org/10.1038/ srep40864.
Dominguez-Bello, M. G., E. K. Costello, M. Contreras, M. Magris, G. Hidalgo, N. Fierer, and R. Knight. 2010. Delivery mode shapes the acquisition and structure of the initial microbiota across multiple body habitats in newborns. Proc. Natl. Acad. Sci. USA 107:1197111975. https://doi.org/10.1073/pnas.1002601107.

Eisler, M. C., M. R. Lee, J. F. Tarlton, G. B. Martin, J. Beddington, J. A. Dungait, H. Greathead, J. Liu, S. Mathew, H. Miller, T. Misselbrook, P. Murray, V. K. Vinod, R. Van Saun, and M. Winter. 2014. Agriculture: Steps to sustainable livestock. Nature 507:32-34.

Etxeberria, U., N. Arias, N. Boqué, M. T. Macarulla, M. P. Portillo, J. A. Martínez, and F. I. Milagro. 2015. Reshaping faecal gut microbiota composition by the intake of trans-resveratrol and quercetin in high-fat sucrose diet-fed rats. J. Nutr. Biochem. 26:651-660. https://doi.org/10.1016/j.jnutbi0.2015.01.002.

Foditsch, C., R. V. V. Pereira, E. K. Ganda, M. S. Gomez, E. C. Marques, T. Santin, and R. C. Bicalho. 2015. Oral administration of Faecalibacterium prausnitzii decreased the incidence of severe diarrhea and related mortality rate and increased weight gain in preweaned dairy heifers. PLoS One 10:e0145485 https://doi. org/10.1371/journal.pone.0145485.

Fries, P., Y. I. Popowych, L. L. Guan, T. Beskorwaynea, A. Potter, L. Babiuk, and P. J. Griebel. 2011a. Mucosal dendritic cell subpopulations in the small intestine of newborn calves. Dev. Comp. Immunol. 35:1040-1051. https://doi.org/10.1016/j.dci.2011.04.003.

Fries, P., Y. I. Popowych, L. L. Guan, and P. J. Griebel. 2011b. Agerelated changes in the distribution and frequency of myeloid and $\mathrm{T}$ cell populations in the small intestine of calves. Cell. Immunol. 271:428-437. https://doi.org/10.1016/j.cellimm.2011.08.012.

Galvão, K. N., J. E. Santos, A. Coscioni, M. Villaseñor, W. M. Sischo, and A. C. Berge. 2005. Effect of feeding live yeast products to calves with failure of passive transfer on performance and patterns of antibiotic resistance in fecal Escherichia coli. Reprod. Nutr. Dev. $45: 427-440$

Godden, S. 2008. Colostrum management for dairy calves. Vet. Clin. North Am. Food Anim. Pract. 24:19-39. https://doi.org/10.1016/j. cvfa.2007.10.005.

Godden, S. M., D. J. Smolenski, M. Donahue, J. M. Oakes, R. Bey, S. Wells, S. Sreevatsan, J. Stabel, and J. Fetrow. 2012. Heat-treated colostrum and reduced morbidity in preweaned dairy calves: Results of a randomized trial and examination of mechanisms of effectiveness. J. Dairy Sci. 95:4029-4040. https://doi.org/10.3168/ jds.2011-5275.

Gruse, J., E. Kanitz, J. M. Weitzel, A. Tuchscherer, T. Stefaniak, and P. Jawor. 2016. Quercetin feeding in newborn dairy calves cannot compensate colostrum deprivation: Study on metabolic, antioxidative and inflammatory traits. PLoS One 11:e0146932. https://doi. org/10.1371/journal.pone.0146932.

Gutzeit, C., G. Magri, and A. Cerutti. 2014. Intestinal IgA production and its role in host-microbe interaction. Immunol. Rev. 260:76-85. https://doi.org/10.1111/imr.12189.

Guzman, C. E., L. T. Bereza-Malcolm, B. De Groef, and A. E. Franks. 2015. Presence of selected methanogens, fibrolytic bacteria, and Proteobacteria in the gastrointestinal tract of neonatal dairy calves from birth to 72 hours. PLoS One 10:e0133048. https://doi. org/10.1371/journal.pone.0133048.

Harris, T. L. 2016. Immunological effects of yeast products on preweaned dairy calves. PhD Thesis. Texas Tech University, Lubbock.

Hooper, L. V., D. R. Littman, and A. J. Macpherson. 2012. Interactions between the microbiota and the immune system. Science 336:1268-1273. https://doi.org/10.1126/science.1223490.

Hugenholtz, P., and G. W. Tyson. 2008. Microbiology: Metagenomics. Nature 455:481-483. https://doi.org/10.1038/455481a.

Jami, E., A. Israel, A. Kotser, and I. Mizrahi. 2013. Exploring the bovine rumen bacterial community from birth to adulthood. ISME J. 7:1069-1079. https://doi.org/10.1038/ismej.2013.2.

Jasarevic, E., C. L. Howerton, C. D. Howard, and T. L. Bale. 2015. Alterations in the vaginal microbiome by maternal stress are associated with metabolic reprogramming of the offspring gut and brain. Endocrinology 156:3265-3276. https://doi.org/10.1210/ en.2015-1177. 
Johansson, M. E., J. M. Larsson, and G. C. Hansson. 2011. The two mucus layers of colon are organized by the MUC2 mucin, whereas the outer layer is a legislator of host-microbial interactions. Proc. Natl. Acad. Sci. USA 108:4659-4665. https://doi.org/10.1073/ pnas.1006451107.

Khachatryan, A. R., D. D. Hancock, T. E. Besser, and D. R. Call. 2004. Role of calf-adapted Escherichia coli in maintenance of antimicrobial drug resistance in dairy calves. Appl. Environ. Microbiol. 70:752-757. https://doi.org/10.1128/AEM.70.2.752-757.2004.

Khan, M. A., D. M. Weary, and M. A. von Keyserlingk. 2011. Invited review: Effects of milk ration on solid feed intake, weaning, and performance in dairy heifers. J. Dairy Sci. 94:1071-1081. https:// doi.org/10.3168/jds.2010-3733.

Klein-Jöbstl, D., E. Schornsteiner, E. Mann, M. Wagner, M. Drillich, and S. Schmitz-Esser. 2014. Pyrosequencing reveals diverse fecal microbiota in Simmental calves during early development. Front. Microbiol. 5:622 https://doi.org/10.3389/fmicb.2014.00622.

Kogut, M. H., and R. J. Arsenault. 2016. Editorial: Gut health: The new paradigm in food animal production. Front. Vet. Sci. 3:71. https://doi.org/10.3389/fvets.2016.00071.

Léger, D. F., N. C. Newby, R. Reid-Smith, N. Anderson, D. L. Pearl, K. D. Lissemore, and D. F. Kelton. 2017. Estimated antimicrobial dispensing frequency and preferences for lactating cow therapy by Ontario dairy veterinarians. Can. Vet. J. 58:26-34.

Liang, G., N. Malmuthuge, H. Bao, P. Stothard, P. J. Griebel, and L. L. Guan. 2016. Transcriptome analysis reveals regional and temporal differences in mucosal immune system development in the small intestine of neonatal calves. BMC Genomics 17:602. https:// doi.org/10.1186/s12864-016-2957-y.

Liang, G., N. Malmuthuge, T. B. McFadden, H. Bao, P. J. Griebel, P. Stothard, and L. L. Guan. 2014. Potential regulatory role of microRNAs in the development of bovine gastrointestinal tract during early life. PLoS One 9:e92592. https://doi.org/10.1371/ journal.pone.0092592.

Lorenz, I., J. Fagan, and S. J. More. 2011. Calf health from birth to weaning. II. Management of diarrhoea in pre-weaned calves. Ir. Vet. J. 64:9 https://doi.org/10.1186/2046-0481-64-9.

Magalhães, V. J., F. Susca, F. S. Lima, A. F. Branco, I. Yoon, and J. E. Santos. 2008. Effect of feeding yeast culture on performance, health, and immunocompetence of dairy calves. J. Dairy Sci. 91:1497-1509. https://doi.org/10.3168/jds.2007-0582.

Malmuthuge, N., Y. Chen, G. Liang, L. A. Goonewardene, and L. L. Guan. 2015. Heat-treated colostrum feeding promotes beneficial bacteria colonization in the small intestine of neonatal calves. J. Dairy Sci. 98:8044-8053. https://doi.org/10.3168/jds.2015-9607.

Malmuthuge, N., P. J. Griebel, and L. L. Guan. 2014. Taxonomic identification of commensal bacteria associated with the mucosa and digesta throughout the gastrointestinal tracts of preweaned calves. Appl. Environ. Microbiol. 80:2021-2028. https://doi.org/10.1128/ AEM.03864-13

Malmuthuge, N., M. Li, P. Fries, P. J. Griebel, and L. L. Guan. 2012. Regional and age dependent changes in gene expression of Tolllike receptors and key antimicrobial defence molecules throughout the gastrointestinal tract of dairy calves. Vet. Immunol. Immunopathol. 146:18-26. https://doi.org/10.1016/j.vetimm.2012.01.010.

Malmuthuge, N. 2016. Role of gut microbiota in neonatal calf gut development. PhD Thesis. University of Alberta, Edmonton, Canada.

Malmuthuge, N., M. Li, L. A. Goonewardene, M. Oba, and L. L. Guan. 2013. Effect of calf starter feeding on gut microbial diversity and expression of genes involved in host immune responses and tight junctions in dairy calves during weaning transition. J. Dairy Sci. 96:3189-3200. https://doi.org/10.3168/jds.2012-6200.

Mayer, M., J. M. Abenthuma, D. Matthesa, M. J. Kleebergera, C. Egeb, J. Hölzel, J. Bauer, and K. Schwaiger. 2012. Development and genetic influence of the rectal bacterial flora of newborn calves. Vet. Microbiol. 161:179-185. https://doi.org/10.1016/j. vetmic.2012.07.023

Maynou, G., A. Bach, and M. Terre. 2017. Feeding of waste milk to Holstein calves affects antimicrobial resistance of Escherichia coli and Pasteurella multocida isolated from fecal and nasal swabs. J. Dairy Sci. 100:2682-2694. https://doi.org/10.3168/jds.2016-11891.

Maynou, G., L. Migura-Garcia, J. Subirats, H. Chester-Jones, D. Ziegler, A. Bach, and M. Terre. 2016. Impact of milk-feeding programs on fecal bacteria population and antimicrobial resistance genes in Escherichia coli isolated from feces in preweaned calves. J. Anim. Sci. 94:593 https://doi.org/10.2527/jam2016-1232.

Meale, S. J., S. Li, P. Azevedo, H. Derakhshani, J. C. Plaizier, E. Khafipour, and M. A. Steele. 2016. Development of ruminal and fecal microbiomes are affected by weaning but not weaning strategy in dairy calves. Front. Microbiol. 7:582 https://doi.org/10.3389/ fmicb.2016.00582.

Mebius, R. E. 2003. Organogenesis of lymphoid tissues. Nat. Rev. Immunol. 3:292-303.

Oikonomou, G., A. G. Teixeira, C. Foditsch, M. L. Bichalho, V. S Machado, and R. C. Bicalho. 2013. Fecal microbial diversity in pre-weaned dairy calves as described by pyrosequencing of metagenomic 16S rDNA. Associations of Faecalibacterium species with health and growth. PLoS One 8:e63157 https://doi.org/10.1371/ journal.pone.0063157.

Oultram, J., E. Phipps, A. G. Teixeira, C. Foditsch, M. L. Bicalho, and V. S. Machado. 2015. Effects of antibiotics (oxytetracycline, florfenicol or tulathromycin) on neonatal calves' faecal microbial diversity. Vet. Rec. 177:598 https://doi.org/10.1136/vr.103320.

Pacchierotti, F., and M. Spanò. 2015. Environmental impact on DNA methylation in the germline: State of the art and gaps of knowledge. BioMed Res. Int. 2015:123484 https://doi. org/10.1155/2015/123484

Pereira, R. V. V., S. Lima, J. D. Siler, C. Foditsch, L. D. Warnick, and R. C. Bicalho. 2016. Ingestion of milk containing very low concentration of antimicrobials: Longitudinal effect on fecal microbiota composition in preweaned calves. PLoS One 11:e0147525 https:// doi.org/10.1371/journal.pone.0147525.

Plummer, E., J. Twin, D. M. Bulach, S. M. Garland, and S. N. Ta brizi. 2015. A comparison of three bioinformatics pipelines for the analysis of preterm gut microbiota using $16 \mathrm{~S}$ rRNA gene sequencing data. J. Proteomics Bioinform. 8:12 https://doi.org/10.4172/ jpb.1000381.

Rakoff-Nahoum, S., J. Paglino, F. Eslami-Varzaneh, S. Edberg, and R. Medzhitov. 2004. Recognition of commensal microflora by toll-like receptors is required for intestinal homeostasis. Cell 118:229-241.

Sartor, R. B., and S. K. Mazmanian. 2012. Intestinal microbes in inflammatory bowel diseases. Am. J. Gastroenterol. 1:15-21. https://doi.org/10.1038/ajgsup.2012.4.

Sharma, R., U. Schumacher, V. Ronaasen, and M. Coates, 1995. Rat intestinal mucosal responses to a microbial flora and different diets. Gut 36:209-214.

Shendure, J., and H. Ji. 2008. Next-generation DNA sequencing. Nat. Biotechnol. 26:1135-1145. https://doi.org/10.1038/nbt1486.

Smith, G. 2015. Antimicrobial decision making for enteric diseases of cattle. Vet. Clin. North Am. Food Anim. Pract. 31:47-60. https:// doi.org/10.1016/j.cvfa.2014.11.004.

Smith, P. M., and W. S. Garrett. 2011. The gut microbiota and mucosal T cells. Front. Microbiol. 2:111 https://doi.org/10.3389/ fmicb.2011.00111

Sommer, F., and F. Bäckhed. 2013. The gut microbiota-Masters of host development and physiology. Nat. Rev. Microbiol. 11:227-238. https://doi.org/10.1038/nrmicr02974.

Stokholm, J., S. Schjorring, C. E. Eskildsen, L. Pedersen, A. L. Bischoff, N. Folsgaard, C. G. Carson, B. L. Chawes, K. Bønnelykke, A Mølgaard, B. Jacobsson, K. A. Krogfelt, and H. Bisgaard. 2014 Antibiotic use during pregnancy alters the commensal vaginal microbiota. Clin. Microbiol. Infect. 20:629-635. https://doi. org/10.1111/1469-0691.12411

Teran, R., E. Mitre, M. Vaca, S. Erazo, G. Oviedo, M. P. Hübner, M. E. Chico, J. J. Mattapallil, Q. Bickle, L. C. Rodrigues, and P. J. Cooper. 2011. Immune system development during early childhood in tropical Latin America: Evidence for the age-dependent down regulation of the innate immune response. Clin. Immunol. 138:299-310. https://doi.org/10.1016/j.clim.2010.12.011. 
Thames, C. H., A. Pruden, R. E. James, P. P. Ray, and K. F. Knowlton. 2012. Excretion of antibiotic resistance genes by dairy calves fed milk replacers with varying doses of antibiotics. Front. Microbiol. 3:139 https://doi.org/10.3389/fmicb.2012.00139.

Tomassini, L. 2015. Rectal microbiota dynamics in preweaned dairy calves depending on colostrum intake, presence of diarrhea, and antibiotic treatment. PhD Thesis. Washington State University, Pullman.

Tringe, S. G., and P. Hugenholtz. 2008. A renaissance for the pioneering 16S rRNA gene. Curr. Opin. Microbiol. 11:442-446. https:// doi.org/10.1016/j.mib.2008.09.011.

Uetake, K. 2013. Newborn calf welfare: A review focusing on mortality rates. Anim. Sci. J. 84:101-105. https://doi.org/10.1111/asj.12019.

Ulluwishewa, D., R. C. Anderson, W. C. McNabb, P. J. Moughan, J. M. Wells, and N. C. Roy. 2011. Regulation of tight junction permeability by intestinal bacteria and dietary components. J. Nutr. 141:769-776. https://doi.org/10.3945/jn.110.135657.

USDA. 2010. Dairy 2007: Heifer Calf Health and Management Practices on US Dairy Operations, 2007. USDA, Animal and Plant Health Inspection Service, Veterinary Services, Center for Epidemiology and Animal Health, Fort Collins, CO.

Uyeno, Y., Y. Sekiguchi, and Y. Kamagata. 2010. rRNA-based analysis to monitor succession of fecal bacterial communities of Holstein calves. Lett. Appl. Microbiol. 51:570-577. https://doi. org/10.1111/j.1472-765X.2010.02937.x.

Xiao, J. X., G. M. Alugongo, R. Chung, S. Z. Dong, S. L. Li, I. Yoon, Z. H. Wu, and Z. J. Cao. 2016. Effects of Saccharomyces cerevisiae fermentation products on dairy calves: Ruminal fermentation, gastrointestinal morphology, and microbial community. J. Dairy Sci. 99:5401-5412. https://doi.org/10.3168/jds.2015-10563.

Xie, G., G. C. Duff, L. W. Hall, J. D. Allen, C. D. Burrows, J. C. Bernal-Rigoli, S. E. Dowd, V. Guerriero, and C. J. Yeoman. 2013 Alteration of digestive tract microbiome in neonatal Holstein bull calves by bacitracin methylene disalicylate treatment and scours. J. Anim. Sci. 91:4984-4990. https://doi.org/10.2527/jas.20136304.

Yasuda, M., C. N. Jenne, L. J. Kennedy, and J. D. Reynolds. 2006. The sheep and cattle Peyer's patch as a site of B-cell development. Vet. Res. 37:401-415.

Yatsunenko, T., F. E. Rey, M. J. Manary, I. Trehan, M. G. Dominguez-Bello, and M. Contreras. 2012. Human gut microbiome viewed across age and geography. Nature 486:222-227. https:// doi.org/10.1038/nature11053.

Zhou, M., Y. Chen, P. J. Griebel, and L. L. Guan. 2014. Methanogen prevalence throughout the gastrointestinal tract of pre-weaned dairy calves. Gut Microbes 5:628-638. https://doi.org/10.4161/19 490976.2014.969649.

Zijlmans, M. A. C., K. Korpela, J. M. Riksen-Walraven, W. M. de Vos, and C. de Weerth. 2015. Maternal prenatal stress is associated with the infant intestinal microbiota. Psychoneuroendocrinology 53:233-245. https://doi.org/10.1016/j.psyneuen.2015.01.006. 